\title{
Optimal Scheduling for a Service Technician Workforce with Time-varying Work Volume and Technician Availability
}

\author{
Dennis Charles Dietz \\ Analytics and Forecasting, CenturyLink, Inc., Boulder, USA
}

Email address:

dennis.dietz@centurylink.com

\section{To cite this article:}

Dennis Charles Dietz. Optimal Scheduling for a Service Technician Workforce with Time-varying Work Volume and Technician Availability. American Journal of Engineering and Technology Management. Vol. 2, No. 6, 2017, pp. 77-82. doi: 10.11648/j.ajetm.20170206.11

Received: May 30, 2017; Accepted: July 5, 2017; Published: November 7, 2017

\begin{abstract}
A practical scheduling method is developed and implemented to determine the optimal allocation of technicians to candidate tour types and start times in a field service environment. Historical data is aggregated to determine a weekly work volume distribution and technician availability profile. These and other quantitative factors populate a mixed integer programming model for determining the distribution of technician tours that will minimize queueing delay in completing service, subject to side constraints on tour type quantities. The approach has been successfully implemented to schedule installation and maintenance technicians at a major telecommunication service provider and could easily be adapted to other operational contexts.
\end{abstract}

Keywords: Scheduling, Mixed Integer Linear Programming, Operations Management

\section{Introduction}

Many commercial enterprises and public agencies employ field service technicians to provide timely on-site service for patrons. When total workforce capacity is consistent with work volume, the response time and appointment window performance for provided services are determined primarily by the efficacy of the employee scheduling process. The scheduling environment is normally characterized by a variable demand pattern and a requirement to assign technicians to "tours" that are constrained by labor rules. Hence, the fundamental problem is to schedule tours such that resulting time-varying staff quantities maximize service quality, or achieve a target service quality at minimum cost.

Efficient management of a technician workforce involves decision making (and supporting modeling and analysis) on three primary time horizons: annual planning, weekly scheduling, and daily execution. Annual planning deals with strategic concerns such as forecasting long-term work volume trends and associated personnel requirements, managing an employee accession and training pipeline, and planning for work volume seasonality. Daily execution encompasses tactical matters such as consideration of schedule change requests, monitoring of schedule compliance and field performance metrics, and responding to unpredicted fluctuations in work volume by offering discretionary time-off or overtime to appropriate technicians. This article focuses on weekly scheduling, which involves confirming forecast work volume and total technician quantities, adjusting for nonproductive activity requirements (estimating technician "availability"), creating a schedule, and then populating the schedule with particular employees based on skills, seniority, and preferences. Specific attention is directed toward the technical task of creating an optimal weekly schedule, which is derived as an optimal quantification of tours by type and start time.

The importance of the personnel scheduling problem is indicated by a large body of relevant literature. Cogent reviews are presented by den Bergh et al. [1] and Brunner [2], and a comprehensive bibliography is provided by Ernst et al. [3]. Reported application areas include retail sales [4, 5], manufacturing [6], transportation [7, 8, 9], health care delivery $[10,11,12]$, and the telecommunications industry $[13,14]$. Solution approaches have incorporated diverse industrial engineering methods such as mathematical programming [15, 16, 17], simulation [18], dynamic programming [19], genetic algorithms [20], and other heuristic procedures [21, 22]. Lesaint et al. [23] document development and deployment of a dynamic scheduler which employs a combination of heuristic search and constraint- 
based reasoning to schedule 40,000 telecommunication service technicians with estimated cost avoidance of $\$ 250$ million per year. In this article, a practical schedule optimization method is developed and implemented to minimize average job completion delay where work volume and technician availability both follow known time-varying profiles.

\section{Model Parameters}

A forecast weekly volume distribution for a typical service area can be accurately constructed by aggregating multiple weeks of historical data. Figures $1 \mathrm{a}$ and $1 \mathrm{~b}$ display distributions of repair and installation service volume across a circular set of 30 -minute intervals $I=\{1,2, \ldots, 336\}$ for products offered within a particular geographical area serviced by a major telecommunication company. Since all service technicians are qualified to perform both types of work, the data can be combined as shown in Figure 1c. The aggregate volume distribution $v_{i}, i \in I$, suggests that, if all technicians were scheduled to work only Monday through Friday, the significant amount of work arriving over the weekend would be backlogged to Monday. Since Monday is already the day with highest volume, substantial job delay would be cascaded throughout the week unless mitigated by overtime. Similarly, if all technicians were scheduled for identical start times, substantial amounts of repair work become would be inaccessible and consequently backlogged to the next day.

a. Repair

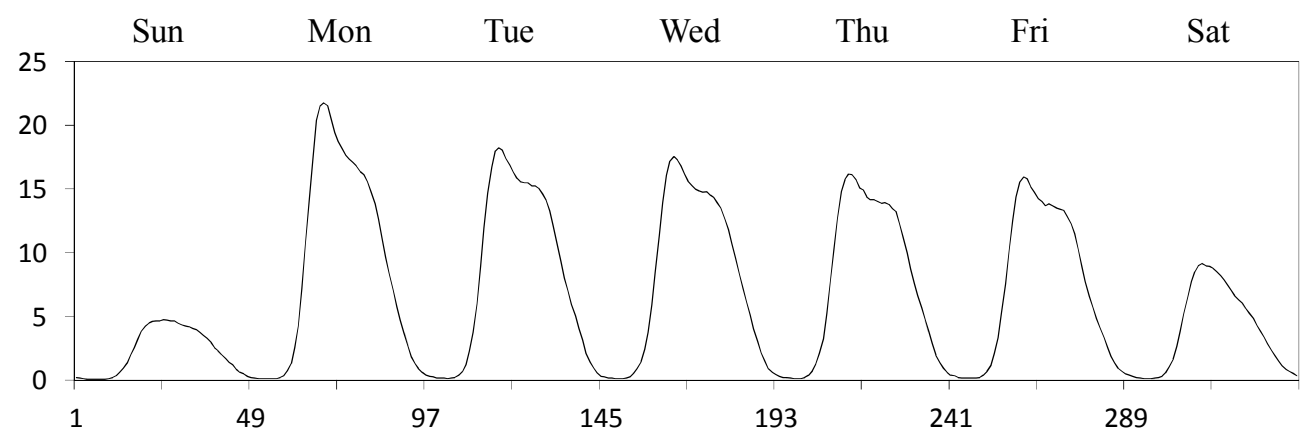

b. Installation

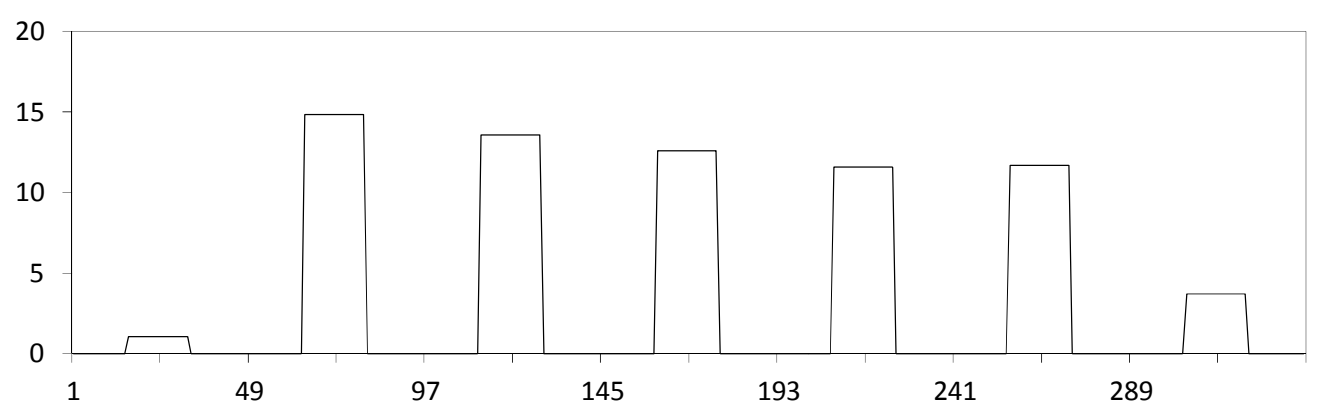

c. Combined

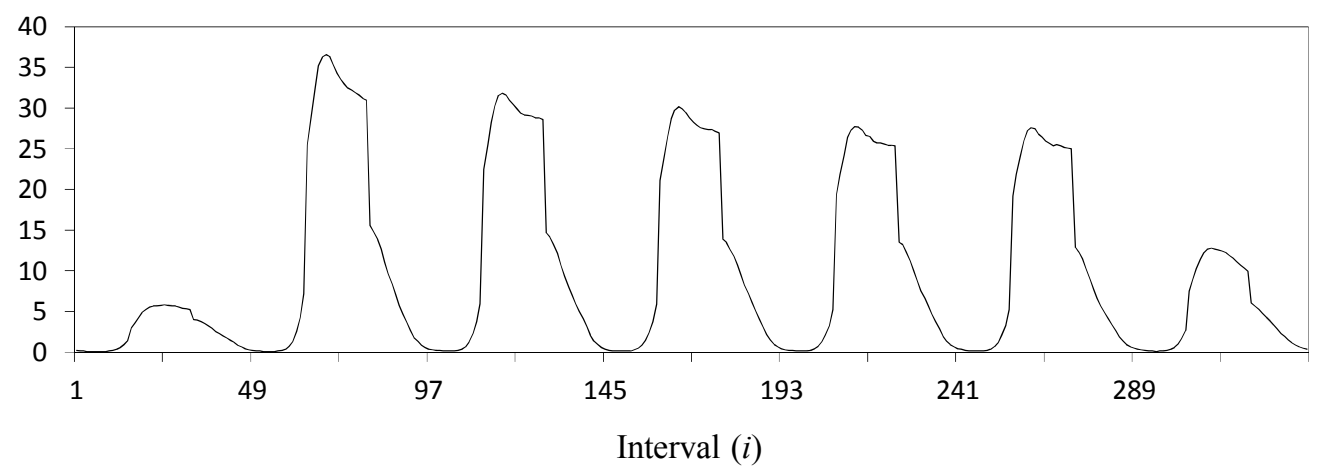

Figure 1. Typical Work Volume Profiles.

Figure 2 displays a representative technician availability profile, which captures interval-dependent variability in the average fraction of time that a scheduled technician is actually available to work in the field after accounting for nonproductive activities such as absences, breaks, meetings, training, and other administrative functions. An approximate availability factor for interval $i$ can be computed as $f_{i} A$, where the profile values $f_{i}$ are aggregated from annual interval data and $A$ is the overall availability estimate for the week. Since the exact technician quantities scheduled on each interval are not initially known, the optimization model must incorporate a normalization process to ensure that overall availability for the optimal schedule equates to $A$. By decoupling $A$ from the associated profile, trends and seasonalities which do not appreciably affect the relative magnitudes across intervals can be conveniently modeled. It 
is noteworthy that the volume and availability profiles must be periodically and simultaneously updated due to interaction between work volume, technician availability, and implemented schedules.

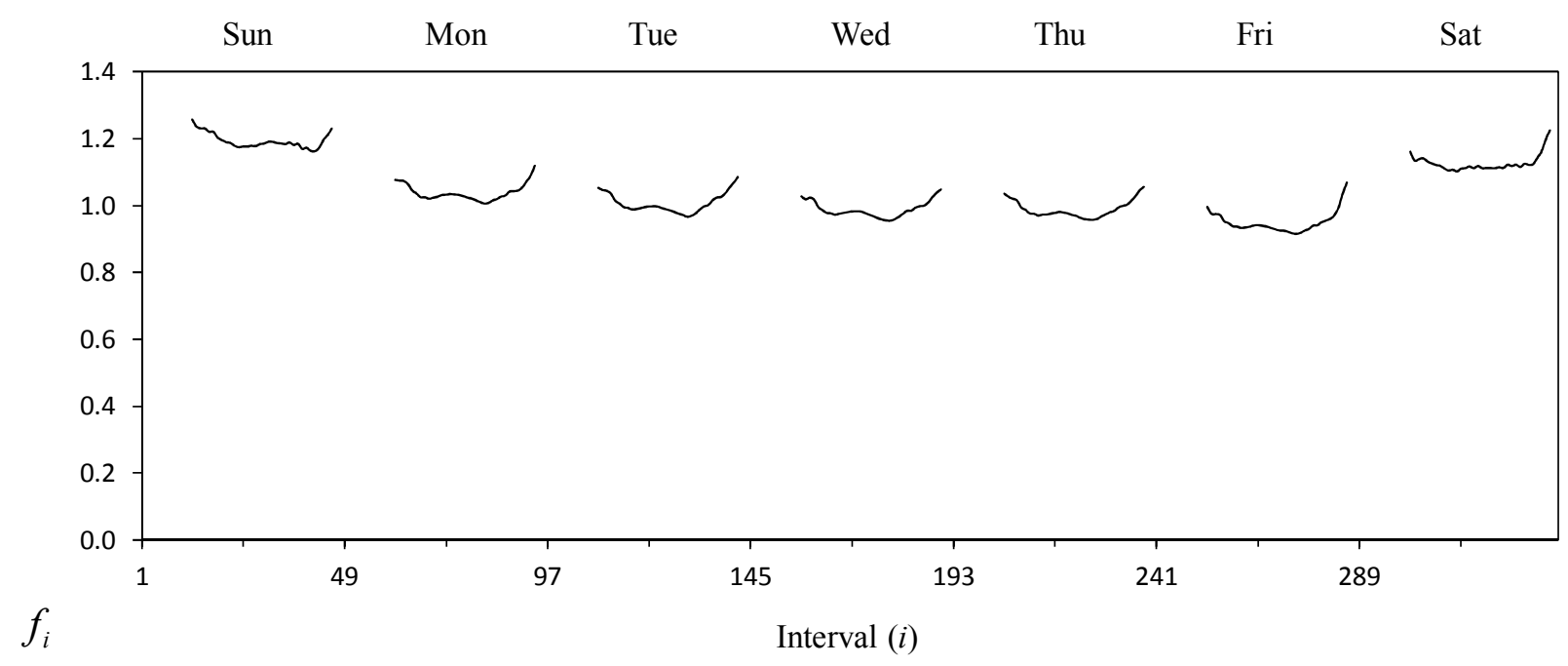

Figure 2. Typical Technician Availability Profile.

\section{Schedule Optimization}

In any given week and geographic area, a specified number of technicians $t$ must be scheduled in shift groupings called "tours" that span multiple intervals. Each tour is characterized by its combination of start time and workday schedule, and each technician is scheduled for five daily shifts per week of 8.5 consecutive hours ( 8 hours of work with 30 minutes for lunch). Eligible start times align with a one-day interval lattice so that specified operational hours are maintained. For operations between 7:30 AM and 6:00 PM (intervals 16 through 37 ), the eligible start times $T_{j}$ would include $\{16,17, \ldots, 20\}$ or any selected subset thereof. A coverage parameter $c_{i j}$ indicates which intervals $i$ are covered by tour type $j$ when artificially assuming a start time of midnight (interval 1). This parameter is generally binary, though we represent the lunch period with a 90-minute notch $(\ldots, 1.00,1.00,0.75,0.50,0.75,1.00,1.00, \ldots)$ rather than a 30 -minute slot $(\ldots, 1.00,1.00,0.00,1.00,1.00, \ldots)$ to acknowledge the likely tactical staggering of lunch periods for technicians scheduled for the same shift. Lunches could be staggered explicitly by introducing more tour types, but the resulting schedules would be far more complex and could create technician expectations that inhibit tactical flexibility in responding to unpredicted fluctuations in workload.

Diversity of the available tours ensures that each technician works five days in each week, Saturdays and Sundays are adequately staffed, and higher volume days employ more technicians. In any feasible schedule, some technicians may be assigned to simple Monday through Friday tours (from the set denoted $S_{5}$ ). Other technicians will be assigned a Saturday or Sunday tour (from the set $S_{1}$ ), along with a weekday tour with an off-day (from the set $S_{4}$ ). Each technician's weekday start time will not vary within the week. However, a technician assigned to an off-day tour may have a different tour type and start time on the assigned
Saturday or Sunday. For notational convenience, we define the weekly number of intervals covered by a scheduled technician as $C=80$, and define a constant $h=30$ minutes as the technician work capacity for each interval.

The modeling objective is to generate a tour distribution that minimizes the total amount of work backlogged. Additional constraints are included to restrict the number of type $j$ tours to values no greater than specified limits $n_{j}$ (for example, Saturday and Sunday tours may be limited due to their higher cost and lower desirability). To capture these requirements, we formulate a mixed integer linear program (MILP) in which each decision variable $x_{j k}$ is the number of technicians assigned to a tour of type $j$ with start time $k \in T_{j}$. Letting auxiliary variables $y_{i}$ be the resulting scheduled staff and $z_{i}$ be the work backlog variables for all intervals $i \in I$, we write the formulation

$$
\operatorname{Minimize} \sum_{i \in I} z_{i}
$$

Subject to

$$
\begin{gathered}
y_{i}=\sum_{j \in S_{5} \cup S_{4}} \sum_{k \in T_{j}} c_{i-k+1, j} x_{j k}, \quad i \in I \\
\sum_{j \in S_{4}} \sum_{k \in T_{j}} x_{j k}=\sum_{j \in S_{1}} \sum_{k \in T_{j}} x_{j k} \\
\sum_{j \in S_{5} \cup S_{4}} \sum_{k \in T_{j}} x_{j k}=t \\
\sum_{k \in T_{j}} x_{j k} \leq n_{j}, \quad j \in S_{5} \cup S_{4} \cup S_{1} \\
\sum_{i \in I} f_{i} y_{i}=C t w \\
z_{i} \geq z_{i-1}+v_{i} w-h A f_{i} y_{i}, \quad i \in I
\end{gathered}
$$




$$
z_{i} \geq 0, \quad i \in I \quad(8) \quad x_{j k} \in\left\{0,1,2, \ldots, n_{j}\right\}, \quad j \in S_{5} \cup S_{4} \cup S_{1}, k \in T_{j}
$$
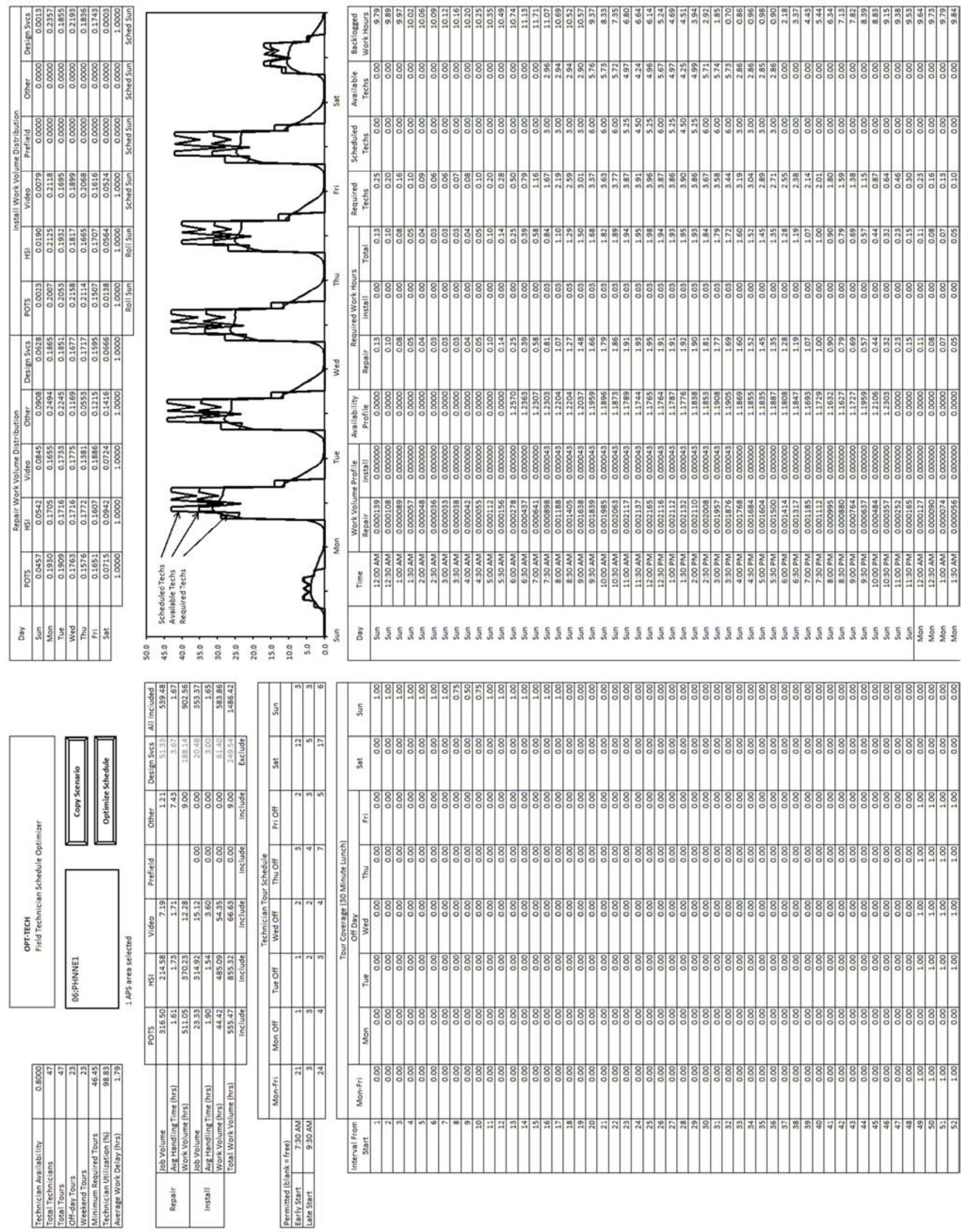

Figure 3. Spreadsheet Implementation of the Scheduling Model. 
Equation 2 defines the relationship between interval staffing levels and scheduled tours, while equation 3 ensures equivalency of off-day and weekend tour quantities. Equation 4 guarantees that exactly $t$ technicians are scheduled, and equation 5 enforces the optional limits on tour type quantities. Equation 6 defines a scaling variable $w$ to correct availability approximation error for realized values of $y_{i}$, and this scaling is applied to the work volume parameters $v_{i}$ in the backlog equation 7. Equations 7 and 8, in conjunction with minimization of the objective function, are functionally equivalent to the backlog defining expression

$$
z_{i}=\max \left\{0, z_{i-1}+v_{i} w-h A f_{i} y_{i}\right\}
$$

The availability correction scaling must be applied to determine true backlog as $z_{i}^{\prime}=z_{i} / w, i \in I$. Applying Little's Law [24], average work delay $D$ can be derived as

$$
D=\frac{\sum_{i \in I} z_{i}^{\prime}}{\sum_{i \in I} v_{i} / h}
$$

\section{Implementation and Results}

The complete method for determining an optimal schedule and predicting performance has been implemented in a Microsoft Excel spreadsheet environment. Figure 3 displays key components of the model, including a schedule matrix, global input parameters and results, a coverage parameter matrix, and interval performance results. Error checking (type and range) is performed on all data inputs. The work volume
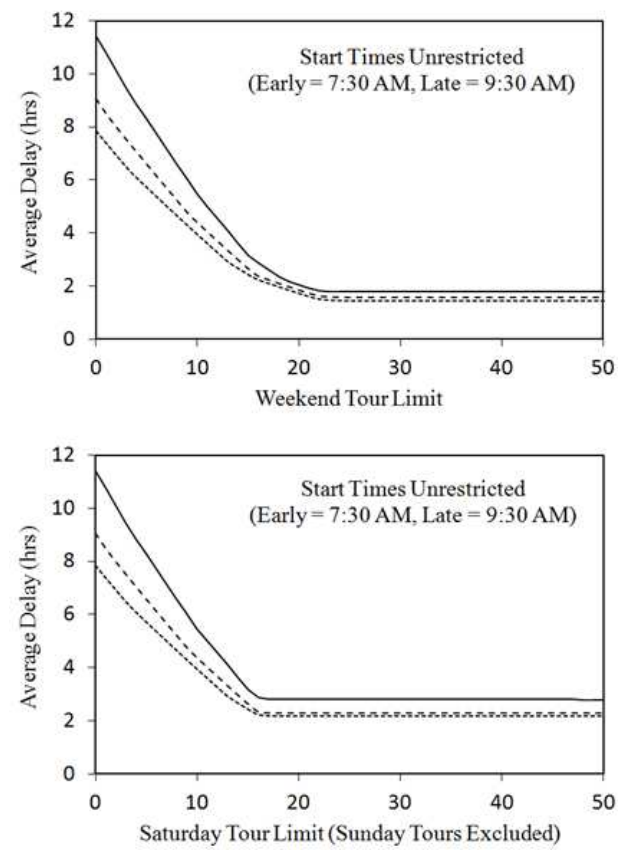

Figure 4. Sensitivity of Average Delay to Tour Diversity.

The scenario modeled in Figure 3 schedules 47 technicians with $80 \%$ overall availability, and yields an average job delay of 1.79 hours. If the same technicians were all scheduled for

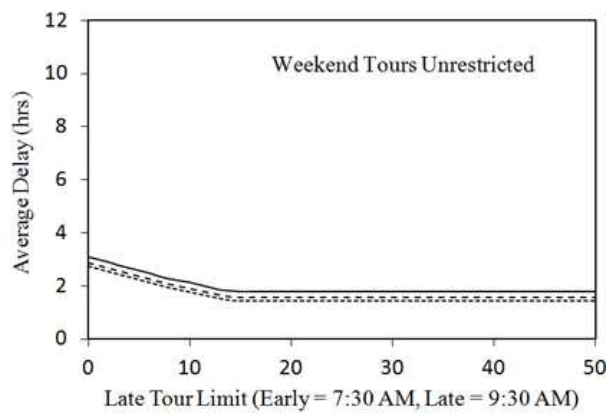

profile is an aggregation of 52 weeks of historical data which can be imported from company systems using an interface ribbon accessible from the Excel main menu. For operational simplicity, only two selectable start times ("early" and "late") are considered in the implemented model. Tours can be entered manually and the model will check for feasibility, alerting the user to discrepancies through warning messages. Consistent with the optimization model formulation, interval staffing levels are computed using equation 2 and the work volume must be scaled to enforce static overall availability for each schedule evaluated. The scaling factor is determined as

$$
w=\frac{\sum_{i \in I} f_{i} y_{i}}{C t}
$$

The available staff on each interval $i$ can then be calculated as

$$
a=A f_{i} y_{i} / w
$$

and backlog is computed as

$$
z_{i}^{\prime}=\max \left\{0, z_{i-1}^{\prime}+v_{i}-h a_{i}\right\}
$$

An optimal schedule can be generated by a single mouse click on the "Optimize Schedule" button. Optimal solutions are obtained using the OpenSolver application [25] with the default MILP algorithm and settings. Large problems with more than 500 technicians solve in less than 5 seconds. Any optimal schedule can be modified manually, and the effect on predicted performance can be immediately observed.

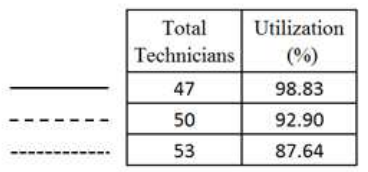

Mon-Fri tours at the early start time (7:30 AM), the average job delay would be 13.19 hours. This seven-fold difference powerfully illustrates the value of schedule optimization. The 
sensitivities of average delay to tour diversity for the same scenario at various levels of technician utilization are illustrated in Figure 4. To isolate various effects, late tours are unconstrained in the left graphs and weekend tours are unconstrained on the right. Weekend scheduling clearly produces much more substantial reduction in average delay than late shift scheduling. When Saturday and Sunday tours are permitted, most of the delay reduction can be obtained from the first $70 \%$ of the optimal tour quantities. When Sunday tours are excluded, the delay reduction from Saturday tour scheduling is nearly linear until the optimal tour quantity is scheduled. These general insights are quite helpful in selecting eligible start times and manually adjusting schedules to accommodate additional operational factors such as daylight, customer preferences, and the availability of staff in supporting organizations.

\section{Conclusions}

Optimization theory and practical considerations have been integrated to create a spreadsheet-based model for field technician scheduling. The model has been successfully implemented by a major telecommunications company, resulting in more efficient workforce utilization, reduced service interval, and improved uniformity in service quality. In some geographic areas, the model has been expanded to accommodate additional tour types and varying operational practices. Hence, cumulative experience suggests that the approach is quite flexible and could be applied to myriad field service environments.

\section{References}

[1] J. V. den Bergh, J. Belien, P. De Bruecker, E. Demeeulemeester, L. De Boeck, Personnel Scheduling: A Literature Review, European Journal of Operational Research 226 (3) (2013) 367-385.

[2] J. O. Brunner, Literature Review on Personnel Scheduling, Flexible Shift Planning in the Service Industry, Lecture Notes in Economics and Mathematical Systems 640 (2010) 5-12.

[3] A. T. Ernst, H. Jiang, M. Krishnamoorthy, B. Owens, D. Sier, An Annotated Bibliography of Personnel Scheduling and Rostering, Annals of Operations Research 127 (2004) 21-144.

[4] A. Parisio, C. N. Jones, A Two-stage Stochastic Programming Approach to Employee Scheduling in Retail Outlets with Uncertain Demand, Omega 53 (2015) 97-103.

[5] S. A. Zolfaghari, A. El-Bouri, B. Namiranian, V. Quan, Heuristics for Large Scale Scheduling in the Retail Sector, INFOR 45 (2007) 111-122.

[6] O. Berman, R. C. Larson, E. Pinker, Scheduling Workforce and Workflow in a High Volume Factory, Management Science 43 (1997) 158-172.

[7] S. C. K. Chu, Generating, Scheduling, and Rostering of Shiftcrew Duties: Applications at the Hong Kong International Airport, European Journal of Operational Research 177 (2007) 1764-1778.
[8] B. Gopalakrishnan, E. L. Johnson, Airline Crew Scheduling: State-of-the-art, Annals of Operations Research 140 (2005) 305-337.

[9] B. M. Smith, A. Wren, A Bus Crew Scheduling System Using a Set Covering Formulation, Transportation Research Part A: General 22 (1988) 97-108.

[10] S. Topalogu, A Multi-objective Programming Model for Scheduling Emergency Medicine Residents, Computers and Industrial Engineering 51 (2006) 375-388.

[11] D. Parr, J. Thompson, Solving the Multi-objective Nurse Scheduling Problem with a Weighted Cost Function, Annals of Operational Research 155 (2007) 279-288.

[12] N. K. Kwak, C. Lee, A Linear Goal Programming Model for Human Resource Allocation in a Health-care Organization, Journal of Medical Systems 21 (1997) 129-140.

[13] D. C. Dietz, Practical Scheduling for Call Center Operations, Omega 39 (2010) 550-557.

[14] M. Segal, The Operator-scheduling Problem: A Network-flow Approach, Operations Research 22 (1974) 808-823.

[15] A. Caprara, M. Monaci, P. Toth, Models and Algorithms for a Staff Scheduling Problem, Mathematical Programming 98 (2003) 445-476.

[16] A. Billionnet, Integer Programming to Schedule a Hierarchical Workforce with Variable Demands, European Journal of Operational Research 114 (1999) 105-114.

[17] G. M. Thompson, Improved Implicit Optimal Modeling of the Labor Shift Scheduling Problem, Management Science 41 (4) (1995) 595-607.

[18] A. P. Muhlemann, A Simulation Study of the Operations of a Telephone Bureau, Omega 9 (2002) 633-637.

[19] M. Elshafei, H. K. Alfares, A Dynamic Programming Algorithm for Days-off Scheduling with Sequence Dependent Labor Costs, Journal of Scheduling 11 (2008) 85-93.

[20] A. Ingolfsson, A. Haque, A. Umnikov, Accounting for Timevarying Queueing Effects in Workforce Scheduling, European Journal of Operational Research 139 (2002) 585-597.

[21] J. Li, E. K. Burke, T. Curtois, S. Petrovic, R. Qu, The Falling Tide Algorithm: A New Multi-objective Approach for Complex Workforce Scheduling, Omega 40 (2012) 283-293.

[22] W. B. Henderson, W. L. Berry, Heuristic Methods for Telephone Operator Shift Scheduling, Management Science 22 (1976) 1372-1380.

[23] D. Lesaint, C. Voudouris, N. Azarmi, Dynamic Workforce Scheduling for British Telecommunications plc, Interfaces 30 (2000) 45-56.

[24] J. D. C. Little, A Proof of the Queuing Formula $L=\lambda W$, Operations Research 9 (1961) 383-387.

[25] A. J. Mason, OpenSolver-An Open Source Add-in to Solve Linear and Integer Programmes in Excel, Operations Research Proceedings 2011, eds. D. Klatte, H. Luthi, K. Schmedders, Springer Berlin Heidelberg (2012) 401-406, http: //opensolver.org. 